\title{
Uniqueness of the inversive plane of order sixty-four
}

\section{Tim Penttila ${ }^{1}$}

Received: 29 May 2021 / Revised: 18 November 2021 / Accepted: 25 January 2022 /

Published online: 9 February 2022

(C) The Author(s) 2022

\section{Abstract}

The uniqueness of the inversive plane of order sixty-four, up to isomorphism, is established. Equivalently, it is shown that every ovoid of $\operatorname{PG}(3,64)$ is an elliptic quadric.

Keywords Inversive planes · Ovoids · Ovals

Mathematics Subject Classification 51E20 $\cdot$ 51E21

\section{Introduction}

An inversive plane is an incidence structure of points and circles such that:

(i) (i) every three distinct points are incident with a unique circle;

(ii) (ii) given two points $P, Q$ and a circle $C$ on $P$ (but not on $Q$ ), there is a unique circle $D$ incident with both $P$ and $Q$ whose only common point with $C$ is $P$;

(iii) (iii) there are at least four points;

(iv) (iv) there is a non-incident point,circle pair; and

(v) (v) every circle is incident with a non-empty set of points. (See [12, pp. 252-253].)

When the inversive plane $\mathbf{I}$ is finite, there is an integer $n \geq 2$, called the order of $\mathbf{I}$ such that $\mathbf{I}$ has $n^{2}+1$ points, $\mathbf{I}$ has $n^{3}+n$ circles and every circle of $\mathbf{I}$ is incident with $n+1$ points of $\mathbf{I}$. In fact, a finite inversive plane of order $n$ is exactly a $3-\left(n^{2}+1, n+1,1\right)$-design ([12, pp. 252-254]).

An ovoid of $\mathrm{PG}(3, q)$ is a set $\Omega$ of $q^{2}+1$ points, no 3 collinear, if $q>2$; if $q=2$, it is a set of 5 points, no 4 coplanar. A secant plane to an ovoid $\Omega$ is a plane meeting $\Omega$ in more than one point. (A tangent plane is a plane meeting $\Omega$ in a unique point.) The incidence structure $\mathbf{I}(\Omega)$ of points of $\Omega$ and plane sections by secant planes is an inversive plane of order $q$ ( $[12,6.1 .2])$. A finite inversive plane is egglike if it is isomorphic to $\mathbf{I}(\Omega)$, for some ovoid $\Omega$ of PG $(3, q)$. All known finite inversive planes are egglike. Moreover, given ovoids $\Omega_{1}, \Omega_{2}$ of $\operatorname{PG}(3, q)$. $\mathbf{I}\left(\Omega_{1}\right)$ is isomorphic to $\mathbf{I}\left(\Omega_{2}\right)$ if and only if there is a collineation $g$ of $\operatorname{PG}(3, q)$ with $\Omega_{1}^{g}=\Omega_{2}$.

Communicated by M. Lavrauw.

Tim Penttila

penttila86@msn.com

1 School of Mathematical Sciences, The University of Adelaide, Adelaide, Australia 
In 1963, Dembowski proved that every inversive plane of even order is egglike [11], and hence has order a power of two.

There are two known families of finite inversive planes: $\mathbf{I}(\Omega)$, where $\Omega$ is an elliptic quadric of PG $(3, q)$, and $\mathbf{I}(\Omega)$, where $\Omega$ is a Tits ovoid of PG(3, $\left.2^{2 e+1}\right), e \geq 1$ [39] (and these are not isomorphic). The inversive planes $\mathbf{I}(\Omega)$, where $\Omega$ is an elliptic quadric of $\mathrm{PG}(3, q)$, are called Miquelian for, by a result of van der Waerden and Smid [41], they are characterised by the Theorem of Miquel [12, 6.1.5]. The bundle theorem is a similar configuration to Miquel's Theorem [12, pp. 255-256]. It is satisfied by every egglike inversive plane [12, 6.1.4]. In 1980, Kahn proved the converse, so: an inversive plane is egglike if and only if it satisfies the bundle theorem [17].

Here we show that every inversive plane of order sixty-four is Miquelian (Corollary 2 in Sect. 4). Previously known results along these lines are : uniqueness of the inversive planes of orders 2, 3, 4 [42], 5 [8,13,37], 7 [14,37], 9 [35] and 16 [1,21,22] and classification of the inversive planes of orders 8 [1,15,29] and 32 [23]. (For order 4, see also [34].)

Ovoids have been used to construct maximal arcs (and thereby partial geometries) [36], unitals [7,19] and generalised quadrangles [12, p. 304], [25, 3.1.2]. Thus our results have consequences for enumerating Buekenhout-Metz unitals, Thas maximal arcs and Tits generalised quadrangles, which we will not dwell on here.

\section{Background results}

An oval of $\mathrm{PG}(2, q)$ is a set of $q+1$ points, no three collinear. A line $l$ is external, tangent, secant to $O$ accordingly as $|l \cap O|$ is 0,1 or 2 . An example of an oval is a nondegenerate conic. Many other ovals are known in characteristic two; see [27] for the most recent survey. A 1955 result of Segre shows that the situation in odd characteristic is in strong contrast to that in characteristic two.

Theorem 1 [31] An oval of $\mathrm{PG}(2, q), q$ odd, is a conic.

Using this result, the same year, Barlotti and Panella independently classified ovoids of $\mathrm{PG}(3, q), q$ odd.

Theorem 2 [2,24] An ovoid of $\mathrm{PG}(3, q), q$ odd, is an elliptic quadric.

Barlotti proved a little more (and Segre proved a slightly stronger result four years later, and gave a far more explicit statement).

Theorem 3 [2, Sect. 3] [33, Theorem V] An ovoid of $\mathrm{PG}(3, q), q$ even, is an elliptic quadric if and only if every secant plane section is a conic.

The best result in this direction is that of Brown from 2000, although we will not need it here.

Theorem 4 [4] An ovoid of $\mathrm{PG}(3, q), q$ even. is an elliptic quadric if and only if some secant plane section is a conic.

Earlier, in 1963, Dembowski had shown that inversive planes of even order arise from ovoids.

Theorem 5 [11] An inversive plane of even order is egglike, and so has order a power of two. 


\section{Hyperovals in PG(2, 64)}

Theorem 6 U.G. Mitchell 1910 [20] The tangent lines to a conic in $\mathrm{PG}(2, q)$, q even, are concurrent.

So the union of a conic of $\mathrm{PG}(2, q), q$ even, and the point of concurrency of its tangent lines is a hyperoval, which is called a regular hyperoval.

By 1957 [32], it had been shown that all hyperovals of PG(2, 2), PG(2, 4) and PG(2, 8) are regular, and irregular hyperovals of $\operatorname{PG}(2, q)$ had been constructed for $q=2^{h}, h=5$ and $h \geq 7$. Segre raised the question of existence of irregular hyperovals in $\mathrm{PG}(2,16)$ and $\mathrm{PG}(2,64)$. The next year, Lunelli and Sce $[18]$ constructed irregular hyperovals in PG $(2,16)$. Nearly four decades passed before the other question was settled [28] by the construction of two irregular hyperovals in $\mathrm{PG}(2,64)$, one with a group of order 60 ; the other with a group of order 15 . The last of the hyperovals in $\mathrm{PG}(2,64)$ was constructed the following year [30]; it has a group of order 12 . The hyperovals with groups of orders 60 and 15 were generalised to the infinite families of Subiaco hyperovals in [10] in 1996. The hyperoval with a group of order 12 was generalised to the infinite family of Adelaide hyperovals in [9] in 2003. In 2019 [40], hyperovals of PG $(2,64)$ were classified by Vandendriessche.

Theorem 7 There are four isomorphism classes of hyperovals in $\mathrm{PG}(2,64)$.

The regular hyperoval gives rise to two ovals, the conic and the point conic. The Subiaco hyperoval with a group of order 60 gives rise to 3 ovals; the Subiaco hyperoval with a group of order 15 gives rise to 6 ovals and the Adelaide hyperoval gives rise to 8 ovals.

Corollary 1 There are nineteen isomorphism classes of ovals in $\mathrm{PG}(2,64)$.

In more detail, the regular hyperoval contains representatives of two isomorphism classes of ovals- the conic and the pointed conic; the Subiaco hyperoval with a group of order 60 contains representatives of three isomorphism classes of ovals, with groups of orders 60 , 12 and 1; the Subiaco hyperoval with a group of order 15 contains representatives of six isomorphism classes of ovals, one with a group of order 15, one with a group of order 3 and four with a group of order 1; and the Adelaide hyperoval contains representatives of eight isomorphism classes of ovals, two with a group of order 12, one with a group of order 3 and five with a group of order 1 .

\section{Ovoids in PG $(3,64)$}

In order to prove this result, we follow the strategy established in [23].

The set of secant plane sections of an ovoid of $\mathrm{PG}(3, q)$ on a tangent line $\ell$ is a called a pencil with carrier $\ell$.

Let $O_{1}$ and $O_{2}$ be ovals of $\mathrm{PG}(2, q), q$ even and let $P$ be a point not on either oval, nor equal to either nucleus. Then $O_{1}$ and $O_{2}$ are compatible at $P$ if they have the same nucleus, they have a point $Q$ in common, the line $P Q$ is a tangent line to each oval and every secant line to $O_{1}$ on $P$ is external to $O_{2}$ (and hence every external line to $O_{1}$ on $P$ is secant to $O_{2}$ ).

In order to keep the treatment as synthetic as possible, we lift some of the ideas of [5].

An augmented fan of ovals of $\operatorname{PG}(2, q)$ is a set $F$ of ovals with common nucleus $N$ and common point $Q$ indexed by the points (other than $P$ ) of a conic $C$ on $P$ with nucleus $N$ so 
that $F=\left\{O_{X}: X \in C, X \neq P\right\}$ such that $O_{X}$ and $O_{Y}$ are compatible at $X Y \cap P N$, for all $X \neq Y \in C \backslash\{P\}$. $C$ is called the augmenting conic of $F$.

Using this terminology, we can restate the Plane Equivalent Theorem without using coordinates.

Theorem 8 [Plane Equivalent Theorem] [23, Theorem 2.1] An ovoid $\Omega$ in $\mathrm{PG}(3, q)$, q even, is equivalent to an augmented fan $\left\{O_{X}: X \in C, X \neq Q\right\}$ of ovals of $\mathrm{PG}(2, q)$. Moreover, for every tangent line $\ell$ each pencil with carrier $\ell$ gives rise to such a set and for each plane $\pi$ of the pencil there is a parameterisation of the planes $\pi_{s}$ of the pencil, $s \in G F(q)$ such that $\pi_{0}=\pi$, a parameterisation of the points $P_{S}$ of the augmenting conic, other than the common point, and there is a homography $M_{s}$ taking $\pi_{s} \cap \Omega$ to $O_{P_{s}}$ and the carrier line of the pencil to the line $Q N$.

We note that the Plane Equivalent Theorem [23, Theorem 2.1] was proved in [26] and [16] (independently), and first published in [21]. (As it was applied, in [5,6,22,23], it was (slightly) modified into a more useful form, and it is in that form that it is stated here, rather than the original form of [26].)

Theorem 9 [23, Theorem 3.1] Let $O$ be an oval of $\mathrm{PG}(2, q)$. If an oval equivalent to $O$ appears as a plane section of an ovoid of $\mathrm{PG}(3, q)$, then for every tangent line $\ell$ of $O$, there is an augmented fan of ovals containing $O$. Furthermore, for every point $P$ on $\ell$ off $O$, not the nucleus of $O$, one of the ovals of this fan is compatible with $O$ at $P$.

Lemma 1 [23, Lemma 3.2] Let $\left(O_{1}, P_{1}\right)$ and $\left(O_{2}, P_{2}\right)$ be two oval-point pairs. If there is a collineation $g$ such that $g P_{2}=P_{1}$ and $g O_{2}$ is compatible with $O_{1}$ at $P_{1}$, then $g$ maps the lines through $P_{2}$ secant to $O_{2}$ to the lines through $P_{1}$ external to $O_{1}$, and the one line through $P_{2}$ tangent to $\mathrm{O}_{2}$ to the one line through $P_{1}$ tangent to $O_{1}$.

Now the lines through any point $P$ can be parameterised by a single non-homogeneous parameter in the following fashion: select two lines, say $\left[a_{1}, b_{1}, c_{1}\right]$ and $\left[a_{2}, b_{2}, c_{2}\right]$ and give them parameters $\infty$ and 0 , respectively. Then the line $\left[\lambda a_{1}+a_{2}, \lambda b_{1}+b_{2}, \lambda c_{1}+c_{2}\right]$ also passes through $P$ and will be labelled $\lambda$. The lines through any point are thus parameterised with $G F(q) \cup \infty$, in the same way as $\operatorname{PG}(1, q)$ is parameterised when $(x, y)$ is given parameter $x / y($ with $1 / 0=\infty)$.

Any collineation $g$ such that $g P_{2}=P_{1}$ maps the lines through $P_{2}$ to the lines through $P_{1}$ and induces an element $g^{\prime} \in P \Gamma L(2, q)$ on the corresponding sets of parameters. Returning to the situation of the two oval-point pairs $\left(O_{1}, P_{1}\right)$ and $\left(O_{2}, P_{2}\right)$ we can insist that the two tangent lines involved both be assigned the parameter $\infty$. The stabiliser of $\infty$ in $P \Gamma L(2, q)$ is the group $A \Gamma L(1, q)$. Therefore a collineation $g$ such that $g P_{2}=P_{1}$ and $g O_{2}$ is compatible with $O_{1}$ at $P_{1}$ induces an element $g^{\prime} \in A \Gamma L(1, q)$ that maps the set of parameters of the external lines to $O_{2}$ through $P_{2}$ to the set of parameters of the secant lines to $O_{1}$ through $P_{1}$. The next theorem provides a converse to this result.

Theorem 10 [23, Theorem 3.3] Let $\left(O_{1}, P_{1}\right)$ and $\left(O_{2}, P_{2}\right)$ be two oval-point pairs, with the lines through $P_{1}$ and $P_{2}$ parameterised in such a way that the tangent lines to the two ovals receive the parameter $\infty$. Let $S_{1}$ and $E_{2}$ be the sets of parameters of the secant lines to $O_{1}$ through $P_{1}$ and the external lines to $O_{2}$ through $P_{2}$, respectively. Then there exists a collineation $g$ such that such that $g P_{2}=P_{1}$ and $g O_{2}$ is compatible with $O_{1}$ at $P_{1}$ if and only if there is an element $g^{\prime} \in A \Gamma L(1, q)$ such that $g^{\prime} E_{2}=S_{1}$. 
Now it is straightforward to see which ovals can appear together in an ovoid. For every ovalpoint pair $(O, P)$, we parameterise the lines through $P$ as described above and determine the local secant parameter set $L$ as the set of parameters of the secant lines. Then two oval-point pairs $\left(O_{1}, P_{1}\right)$ and $\left(O_{2}, P_{2}\right)$ with local secant parameter sets $L_{1}$ and $L_{2}$ are said to match provided that there is some element $g^{\prime} \in A \Gamma L(1, q)$ such that $g^{\prime} L_{1}=\left\{x \in G F(q): x \notin L_{2}\right\}$. Two ovals can only appear together in the same pencil of an ovoid if they both have points at which the corresponding two oval-point pairs match.

\section{The computational results}

For each of the 19 ovals of PG(2, 64), the group stabilising each oval was computed, along with the orbits of this stabiliser on non-nucleus points off the ovals, and a list of all possible oval-point pairs was made.

The conic has a group of order 1,572,480 which has one orbit on points not on the hyperoval containing the conic; the pointed conic has a group of order 24,192 which has two orbits on points not on the hyperoval containing the pointed conic; the oval $O$ with a group of order 60 has 76 orbits on points not on the hyperoval containing $O$; the oval $O$ with a group of order 15 has 275 orbits on points not on the hyperoval containing $O$; the three ovals $O$ with a group of order 12 have 351 orbits on points not on the hyperoval containing $O$; the two ovals $O$ with groups of order 3 have 1375 orbits on points not on the hyperoval containing $O$; the ten ovals $O$ with groups of order 1 have 4095 orbits on points not on the hyperoval containing $O$.

After this computation, there remains a list of 45107 oval-point pairs. For each oval-point pair, the local secant parameter set $L$ was computed, and a canonical representative of the orbit of $L$ under $A \Gamma L(1,64)$ was stored (the representative was the lexicographically least local secant parameter set in the orbit). For each oval-point pair, a canonical representative of the orbit of the complement in $G F(64)$ of $L$ was also stored. Two oval-point pairs match if and only if the canonical representative of the local secant parameter set of the first is actually equal to the canonical representative of the complement of the local secant parameter set of the second. All possible matches between oval-point pairs were thus determined.

Of the 19 ovals, 18 had points at which they do not match with anything at all; hence, none of these 18 ovals can be a secant plane section of an ovoid. The remaining oval is a conic. Hence, every plane section of an ovoid of $\mathrm{PG}(3,64)$ is a conic.

Theorem 11 Every ovoid of $\mathrm{PG}(3,64)$ is an elliptic quadric.

Proof This follows from the results above and Theorem 3.

\section{Consequences}

Theorem 12 Every inversive plane of order 64 is Miquelian.

Proof This follows from Theorems 11 and 5.

Corollary 2 There is a unique inversive plane of order 64.

Proof This follows from the result of van der Waerden and Smid mentioned in the introduction and the fact that any two elliptic quadrics of $\mathrm{PG}(3, q)$ are equivalent under a collineation of $\mathrm{PG}(3, q)$. 
Corollary 3 There is a unique $3-(4097,65,1)$ design.

Proof This is a reformulation of Corollary 2 in the language of design theory.

Corollary 4 A symplectic translation plane of order 4096 with kernel containing GF(64) is Desarguesian.

Proof By applying Theorem 11 and [33, Theorem III], it follows that a symplectic spread of $\mathrm{PG}(3,64)$ is regular, since the symplectic generalised quadrangle $W(q)$ is self-dual $[25$, 3.2.1] in characteristic 2 .

A point $P$ of a generalised quadrangle of order $(s, t)$ is 3-regular if

$$
\left|\{P, Q, R\}^{\perp \perp}\right|=s+1,
$$

whenever $Q$ and $R$ are points that are not collinear, and both not collinear with $P$. The points and lines contained in an elliptic quadric of $\mathrm{PG}(5, q)$ form a generalised quadrangle of order $\left(q, q^{2}\right)$, called $Q(5, q)$ [25, Sect. 3.1]. Our work yields two characterisations of this generalised quadrangle for $q=64$.

Corollary 5 A generalised quadrangle of order $(64,4096)$ with a 3-regular point is isomorphic to $Q(5,64)$.

Proof This is a consequence of Theorem 11 and [25, 5.3.1,3.2.4].

Corollary 6 A generalised quadrangle of order $(64,4096)$ with a subquadrangle isomorphic to $W(64)$ is isomorphic to $Q(5,64)$.

Proof This is a consequence of Theorem 11 and [38, Theorem 7.1].

A generalised quadrangle has Property (G) at a pair $\left\{P, P^{\prime}\right\}$ of collinear points if, every triple $\{P, Q, R\}$ of points, with $P, Q, R$ pairwise non-collinear and $P^{\prime} \in\{P, Q, R\}^{\perp}$, is 3-regular.

Corollary 7 A generalised quadrangle of order $(64,4096)$ that satisfies Property $(G)$ at a pair of distinct collinear points is the dual of a flock generalised quadrangle.

Proof This is a consequence of Theorem 11 and [3, Theorem 25].

Acknowledgements I thank Alessandro Siciliano for checking my calculations from Sect. 5 with Magma.

Funding Open Access funding enabled and organized by CAUL and its Member Institutions

Open Access This article is licensed under a Creative Commons Attribution 4.0 International License, which permits use, sharing, adaptation, distribution and reproduction in any medium or format, as long as you give appropriate credit to the original author(s) and the source, provide a link to the Creative Commons licence, and indicate if changes were made. The images or other third party material in this article are included in the article's Creative Commons licence, unless indicated otherwise in a credit line to the material. If material is not included in the article's Creative Commons licence and your intended use is not permitted by statutory regulation or exceeds the permitted use, you will need to obtain permission directly from the copyright holder. To view a copy of this licence, visit http://creativecommons.org/licenses/by/4.0/. 


\section{References}

1. Ball S.: On ovoids of PG(3, q). Adv. Geom. 6, 177-198 (2006).

2. Barlotti A.: Un'estensione del teorema di Segre-Kustaanheimo. Bollettino dell'Unione Matematica Italiana 10, 498-506 (1955).

3. Barwick S.G., Brown M.R., Penttila T.: Flock generalized quadrangles and tetradic sets of elliptic quadrics of PG(3, q). J. Comb. Theory Ser. A 113, 273-290 (2006).

4. Brown M.R.: Ovoids of PG(3, $q), q$ even, with a conic section. J. Lond. Math. Soc. 2(62), 569-582 (2000).

5. Brown M.R., O’Keefe C.M., Payne S.E., Penttila T., Royle G.F.: Spreads of $T_{2}(O)$, $\alpha$-flocks and ovals. Des. Codes Cryptogr. 31, 251-282 (2004).

6. Brown M., O’Keefe C.M., Payne S.E., Penttila T., Royle G.F.: The classification of spreads of $T_{2}(O)$ and $\alpha$-flocks over small fields. Innov. Incidence Geom. 6, 111-126 (2008).

7. Buekenhout F.: Existence of unitals in finite translation planes of order $q^{2}$ with a kernel of order $q$. Geom. Dedicata 5, 189-194 (1976).

8. Chen Y.: The Steiner system $S(3,6,26)$. J. Geom. 2, 7-28 (1972).

9. Cherowitzo W., O'Keefe C.M., Penttila T.: Unified construction of finite geometries associated with q-clans in characteristic two. Adv. Geom. 3, 1-21 (2003).

10. Cherowitzo W., Penttila T., Pinneri I., Royle G.F.: Flocks and ovals. Geom. Dedicata 60, 17-37 (1996).

11. Dembowski P.: Inversive planes of even order. Bull. Am. Math. Soc. 69, 850-854 (1963).

12. Dembowski P.: Finite Geometries. Springer, Berlin (1968), reprinted (1997).

13. Denniston R.H.F.: Uniqueness of the inverse plane of order 5. Manuscripta Math. 8, 11-19 (1973).

14. Denniston R.H.F.: Uniqueness of the inversive plane of order 7. Manuscripta Math. 8, 21-26 (1973).

15. Fellegara G.: Gli ovaloidi in uno spazio tridimensionale di Galois di ordine 8. Atti Accad. Naz. Lincei Rend. Cl. Sci. Fis. Mat. Nat. 32, 170-176 (1962).

16. Glynn D.G.: Plane representations of ovoids. Bull. Belgian Math. Soc. 5, 275-286 (1998).

17. Kahn J.: Inversive planes satisfying the bundle theorem. J. Comb. Theory Ser. A 79, 1-19 (1980).

18. Lunelli L., Sce M.: $k$-archi completi nei piani proiettivi desarguesiani di rango 8 e 16 . Centro di Calcoli Numerici, Politecnico di Milano (1958).

19. Metz R.: On a class of unitals. Geom. Dedicata 8, 125-126 (1979).

20. Mitchell U.G.: Geometry and collineation groups of the finite projective Plane PG(2, $\left.2^{2}\right)$, Ph. D. thesis, Princeton (1910).

21. O'Keefe C.M., Penttila T.: Ovoids of PG(3, 16) are elliptic quadrics. J. Geom. 38, 95-106 (1990).

22. O'Keefe C.M., Penttila T.: Ovoids of PG(3, 16) are elliptic quadrics II. J. Geom. 44, 140-159 (1992).

23. O'Keefe C.M., Penttila T., Royle G.F.: Classification of ovoids in PG(3, 32). J. Geom. 50, 143-150 (1994).

24. Panella G.: Caratterizzazione delle quadriche di uno spazio (tridimensionale) lineare sopra un corpo finito. Boll. Un. Mat. Ital. 3(10), 507-513 (1955).

25. Payne S.E., Thas J.A.: Finite generalized quadrangles. Research Notes in Mathematics, 110. Pitman (Advanced Publishing Program), Boston, MA, 1984. Second edition. EMS Series of Lectures in Mathematics. European Mathematical Society (EMS), Zürich (2009).

26. Penttila T.: A plane representation of ovoids. Utilitas Math. 56, 245-250 (1999).

27. Penttila T.: Configurations of ovals, Combinatorics, 2002 (Maratea). J. Geom. 76, 233-255 (2003).

28. Penttila T., Pinneri I.: Irregular hyperovals in PG(2, 64). J. Geom. 51, 89-100 (1994).

29. Penttila T., Praeger C.E.: Ovoids and translation ovals. J. Lond. Math. Soc. 2(56), 607-624 (1997).

30. Penttila T., Royle G.F.: On hyperovals in small projective planes. J. Geom. 54, 91-104 (1995).

31. Segre B.: Ovals in a finite projective plane. Can. J. Math. 7, 414-416 (1955).

32. Segre B.: Sui k-archi nei piani finiti di caratteristica due. Rev. Math. Pures Appl. 2, 289-300 (1957).

33. Segre B.: On complete caps and ovaloids in three-dimensional Galois spaces of characteristic two. Acta Arith. 5, 315-332 (1959).

34. Seiden E.: A theorem in finite projective geometry and an application to statistics. Proc. Am. Math. Soc. 1, 282-286 (1950).

35. Steinke G.F.: A remark on Benz planes of order 9. Ars Comb. 34, 257-267 (1992).

36. Thas J.A.: Construction of maximal arcs and partial geometries. Geom. Dedicata 3, 61-64 (1974).

37. Thas J.A.: The affine plane $\mathrm{AG}(2, q), q$ odd, has a unique one point extension. Inventiones Mathematicae 118, 133-139 (1994).

38. Thas J.A., Payne S.E.: Spreads and ovoids in finite generalized quadrangles. Geom. Dedicata 52, 227-253 (1994).

39. Tits J.: Ovoïdes et groupes de Suzuki. Arch. Math. 13, 187-198 (1962).

40. Vandendriessche P.: Classification of the Hyperovals in PG(2, 64). Electron. J. Comb. 26, P2-35 (2019). 
41. van der Waerden B.L., Smid L.J.: Eine Axiomatik der Kreisgeometrie und der Laguerregeometrie. Math. Ann. 110, 753-776 (1935).

42. Witt E.: Über Steinersche Systeme. Abh. Math. Semin. Hansische Univ. 12, 265-275 (1938).

Publisher's Note Springer Nature remains neutral with regard to jurisdictional claims in published maps and institutional affiliations. 